\title{
Intuition als philosophische Methode
}

In der zeitgenössischen analytischen Philosophie wird seit einiger Zeit eine Debatte über den Status der Intuition geführt. Der Ursprung dieser Debatte liegt in den 196oer-Jahren, bei Paul Gettiers Kritik am klassischen Wissensbegriff und der Vielzahl an philosophischen Publikationen, die sich in den folgenden Dekaden mit Gettiers Kritik auseinandergesetzt haben. ${ }^{329}$ Die hier vorliegende Studie reiht sich nicht in die aktuell vorherrschende metaphilosophische Intuitionsdiskussion ein. Vielmehr sollte einen Schritt zurückgetreten und die Intuition grundlegender als Erkenntnisart untersucht werden. Die daraus gewonnenen Erkenntnisse können jedoch auch auf die philosophiemethodische Intuitionsdebatte angewandt werden. Erste Schritte in diese Richtung werden im folgenden Kapitel unternommen.

Zunächst soll in die Grundzüge der Debatte eingeführt werden. Nach verbreiteter Ansicht ist Edmund Gettiers Arbeit ein Paradebeispiel für den erfolgreichen Einsatz der Intuition als philosophische Methode. Gemäß dem auf Platon zurückgehenden klassischen Verständnis ist eine wahre, gerechtfertigte Überzeugung Wissen. Gettier erstellt eine Reihe einfach verständlicher Beispiele, welche dieses Verständnis in Frage stellen: In diesen Beispielen sind die genannten Kriterien gegeben, ohne dass die jeweiligen Subjekte über Wissen verfügen. Hier kommt nun die Intuition ins Spiel: Es kann nach dem Lesen bzw. Hören der Beispiele intuitiv erkannt werden, dass die Subjekte über kein Wissen verfügen. Demnach erscheint dies als unmittelbar und gewiss, ohne dass weitere Gründe für diesen Schluss angeführt werden müssen. Ausgehend von der Diskussion über Gettier wurden viele andere Arbeiten entsprechend eingeordnet bzw. neu entstehende Arbeiten durch diese Methodik inspiriert. ${ }^{330}$ Diesen Arbeiten ist gemeinsam, dass eine bestehende These oder Theorie durch den gezielten Einsatz eines Gegenbeispiels oder eines ausführlicheren, möglicherweise kontrafaktischen Szenarios bzw. Gedankenexperiments in Frage gestellt wird, wobei intuitiv ersichtlich werden soll, dass dieses Gegenbeispiel die Theorie oder These falsifiziert. ${ }^{331}$

329 Siehe Gettier (1963); vgl. Nagel (2007, S. 796f.).

330 Für eine Auflistung weiterer Beispiele siehe Jenkins Ichikawa (2014, S. 232).

331 Dieses Vorgehen wurde selbstverständlich nicht erst mit Gettier in die philosophische Methodik eingeführt; man denke etwa an Platons Dialoge. 
An einem solchen Vorgehen wurde in jüngerer Zeit zunehmend Kritik geäußert. Als Quellen dieser Kritik lassen sich Stephen Stichs Infragestellung der Methoden der analytischen Erkenntnistheorie und die darauf fußenden ersten Arbeiten der sogenannten experimentellen Philosophie (vor allem durch Jonathan Weinberg und Mitarbeiter) benennen. ${ }^{332}$ Sich auf Intuitionen zu berufen wurde in den Augen dieser Kritiker zum hervorstechenden Merkmal einer fehlgeleiteten »Lehnstuhlphilosophie«, welche die psychologische Fundierung und somit Kontingenz der Intuition verkennt. Von der Gegenseite wurde das Verständnis der Intuition als grundlegende philosophische Methode verteidigt, wobei sich die Positionen der Intuitionsverteidigenden deutlich voneinander unterscheiden. Außerdem hat sich in den letzten Jahren drittes Lager in der philosophiemethodischen Intuitionsdebatte formiert, welches sowohl die Lehnstuhl- als auch die experimentellen Philosophinnen und Philosophen dafür kritisiert, dass sie der Intuition als philosophische Methode unbotmäßiges Gewicht geben. Dieses intuitionskritische Lager wird von Timothy Williamson, Herman Cappelen und Max Deutsch angeführt. ${ }^{333}$ Sie halten die Analysen, die in Gettiers und ähnlichen philosophischen Arbeiten eine Berufung auf die Intuition feststellen, für falsch. Im Gegensatz zu Stich und Weinberg sieht das dritte Lager folglich kein Problem in der Kontingenz der Intuition.

Im Folgenden sollen die zentralen Argumente der genannten drei Lager dargestellt und beurteilt werden. Grundlage der Beurteilung ist das in der vorliegenden Arbeit entwickelte Intuitionsverständnis. Nicht alle kritischen Einschätzungen der Argumente sind unabhängig davon gültig, da sich das Intuitionsverständnis der evaluierten Positionen von dem hier erarbeiteten teilweise stark unterscheidet. So sind die folgenden Ausführungen als Verortung des bereits vorausgesetzten neuen Intuitionsverständnisses innerhalb der philosophiemethodischen Debatte zu verstehen. Daraus ergibt sich die Möglichkeit, neue Lösungsansätze zum philosophiemethodischen Problem der Intuition anzudeuten.

\section{1 $»$ Lehnstuhlphilosophie«: Die Intuitionsverteidiger}

Auf der Seite der Verteidiger der Intuition als zentrale philosophische Methode sollen drei einflussreiche Positionen herausgegriffen und in ihren Grundzügen dargestellt werden: diejenigen Alvin Goldmans, George Bealers und Hilary

332 Siehe Stich (1990, Kap. 4.6), Weinberg et al. (2001).

333 Siehe Williamson (2004, 2007), Cappelen (2012), Deutsch (2015). 
Kornbliths. ${ }^{34} \mathrm{Im}$ Vergleich dieser Positionen untereinander zeigt sich, dass »Lehnstuhlphilosophien« trotz ihrer gemeinsamen Auffassung der Intuition als zentrale philosophische Methode diese sehr unterschiedlich interpretieren können. Im Anschluss an die Darstellung wird die jeweilige Position im Hinblick auf das Intuitionsverständnis der vorliegenden Arbeit reflektiert.

\subsubsection{Goldman: Mentalismus}

Alvin Goldman räumt der Intuition innerhalb der Philosophie eine methodisch zentrale Rolle ein. Philosophie besteht Goldman zufolge zu einem wesentlichen Teil aus begriffsanalytischer Arbeit, welche auf der Befragung der Intuition aufbaut. Die methodische Fundierung durch die Intuition ist zugleich ein wichtiges Abgrenzungsmerkmal der Philosophie gegenüber den Naturwissenschaften. ${ }^{335}$ Goldman unterscheidet zwischen mentalistischen und extramentalistischen Ansätzen in Bezug auf die Intuition als philosophische Methode. Seine eigene Position bezeichnet er als mentalistisch. Dies bedeutet, dass uns die Analyse der Intuition eines Subjekts den Zugang zu dessen Konzepten bzw. Verwendungsweisen dieser Konzepte gibt und nicht primär zu Gegenständen der außermentalen Welt. ${ }^{336}$

Die Analyse der Intuition soll nicht nur über subjektive mentale Inhalte Auskunft geben, sondern über allgemeine Begriffe. Dies wird dadurch ermöglicht, dass Intuitionen intersubjektiv geteilt werden. Goldman und Pust exemplifizieren den mentalistischen Lösungsweg am Begriff der Rechtfertigung:

The mentalist does offer an account of what makes a belief justified. The mentalist claims that what makes a belief justified is possession of those properties by virtue of which a deployer of the justification concept, who is aware of all relevant properties of the target belief, would classify it as justified. An account of exactly what those properties are is the very thing that a developed mentalist proposal will provide. ${ }^{337}$

Goldman und Pust postulieren also, dass Intuitionen, wie sie etwa durch Beispiele oder Gedankenexperimente evoziert werden, zur Klärung von Begriffen und somit zur Beantwortung zentraler philosophischer Fragen führen.

334 Einen wichtigen Referenzpunkt für die philosophiemethodische Debatte bildet der Sammelband Rethinking Intuition: siehe DePaul/Ramsey (1998). In diesem Sammelband sind Beiträge der drei genannten Philosophen enthalten. Ein weiterer einflussreicher Intuitionsverteidiger ist Ernest Sosa, von dem ebenfalls ein Beitrag im Sammelband enthalten ist, siehe Sosa (1998).

335 Vgl. Goldman (2007, S. 1).

336 Vgl. Goldman/Pust (1998, S. 187f.).

337 Goldman/Pust (1998, S. 195). 


\title{
6.1.2 Bealer: Sui-Generismus
}

George Bealers Intuitionsverständnis wurde bereits dargestellt (Kap. 3.6). Im Zentrum stand dabei Bealers sui generis-Verständnis der Intuition, wonach Intuitionen Erscheinungen sind, die sich nicht auf andere mentale Zustände zurückführen lassen. Bealers Argumentation in Bezug auf die Abgrenzung der Intuition von Überzeugungen, die sich aus seinem sui generis-Verständnis ergibt, erwies sich als überzeugend, während die Konzeption der Intuition als intellektuelle Erscheinung problematisch blieb.

Im Rahmen der philosophiemethodischen Debatte teilt Bealer mit Goldman die Ansicht, dass Intuitionen für die Philosophie von zentraler Bedeutung sind. Im Gegensatz zu Goldman vertritt Bealer jedoch einen Extramentalismus: Bealer zufolge sind Intuitionen nicht primär Indikatoren unserer Begriffe, sondern gewähren direkten Zugang zur Erkenntnis von Sachverhalten. So soll beispielsweise aus der Intuition, wonach Zombies - also physische Duplikate einer Person, die aber kein Bewusstsein haben - möglich sind, $a$ priori folgen, dass der Physikalismus in der Philosophie des Geistes falsch ist. ${ }^{338}$ Die erkenntnistheoretische Einstellung, welche die Basis dieser Prägung des Extramentalismus bildet, bezeichnet Bealer als >modalen Reliabilismus $<$ :

\begin{abstract}
A general theory of basic evidence must be reliabilist. Contingent reliabilism, however, is beset with fatal problems. To avoid them, we are forced to modal reliabilism: a candidate source of evidence is basic iff its deliverances have an appropriate kind of strong modal tie to the truth. Phenomenal experience is a basic source because it has that kind of modal tie to the truth. ${ }^{339}$
\end{abstract}

Bealer argumentiert weiter, dass die Intuition die Anforderung des modalen Reliabilismus an eine basale Quelle der Evidenz erfüllt. Dies ist Bealer zufolge ebenfalls intuitiv erkennbar:

Intuitively, intuition is a basic source of evidence. For example, suppose a person has an intuition, say, that if $\mathrm{P}$ then not not $\mathrm{P}[\ldots]$. Nothing more is needed. Intuitively, these intuitions are evidentially as basic as evidence gets. They are intuitively as basic as experiences, much as tactile experiences are intuitively as basic as visual experiences. ${ }^{340}$

Was zunächst wie ein problematischer Regress erscheint - mit Intuition wird gerechtfertigt, dass Intuition eine basale Evidenzquelle ist - erweist sich

338 Vgl. Chalmers (1996, S. 94 ff.).

339 Bealer (1998, S. 217).

340 Bealer (1998, S. 217). 
angesichts dieser These als unproblematisch. Gerade wenn die These zutrifft, muss man sich zwangsläufig auf Intuition berufen um sie zu bestätigen.

\title{
6.1.3 Kornblith: Naturalismus
}

Hilary Kornblith ist wie Bealer ein Extramentalist; und wie Bealer und Goldman weist Kornblith der Intuition einen bedeutenden Platz innerhalb der philosophischen Methodik zu. Aufgrund von Kornbliths naturalistisch orientierter Erkenntnistheorie ist dieser Stellenwert jedoch weniger hoch als in den rationalistischen Ansätzen Bealers und Goldmans. Vorrangig soll Intuition als nützliches Instrument im Entdeckungszusammenhang dienen, das in späteren Schritten durch zuverlässigere Methoden ersetzt wird. ${ }^{341} \mathrm{Im}$ Gegensatz zur traditionellen Auffassung versteht Kornblith Intuition als Erkenntnisquelle a posteriori, die sich auf natural kinds bezieht. ${ }^{342}$ Wenn wir also beispielweise in den Gettier-Fällen unsere Intuitionen zur Frage abrufen, ob hier von `Wissen< die Rede sein kann, betrachten wir weder primär unsere eigenen mentalen Zustände noch apriorische Notwendigkeiten, sondern wir verhalten uns ähnlich wie eine Naturwissenschaftlerin, die beurteilt, ob bestimmte Untersuchungsgegenstände unter gegebene natural kinds fallen:

\begin{abstract}
When we appeal to intuitions about knowledge, we make salient certain instances of the phenomenon that need to be accounted for, and that these are genuine instances of knowledge is simply obvious, at least if our examples are well chosen. What we are doing, as I see it, is much like the rock collector who gathers samples of some interesting kind of stone for the purpose of figuring out what it is that the samples have in common. ${ }^{343}$
\end{abstract}

Kornbliths Intuitionsverständnis ist in eine naturalistische Epistemologie eingebettet, deren zentrales Postulat die Verankerung der philosophischen Terminologie in der Welt ist. ${ }^{344}$ Diese Position ist höchst kontrovers. So wendet Goldman gegen Kornblith ein, dass Naturwissenschaftlerinnen im Unterschied zu Philosophinnen einen großen Unterschied zwischen möglichen und realen Beispielen machen:

They devote great time and labor into investigating actual-world objects; they construct expensive equipment to perform their investigations. If the job could be done as well by consulting intuitions about imaginary examples, why bother with all this expensive equipment and labor-intensive experiments? Evidently,

341 Vgl. Kornblith (1998, S. 134f.).

342 Vgl. Kornblith (1998, S. 134).

343 Kornblith (1998, S. 133f.).

344 Vgl. Kornblith (1998, S. 139). 
unless philosophers are either grossly deluded or have a magical shortcut that has eluded scientists (neither of which is plausible), their philosophical inquiries must have a different type of target or subject-matter. ${ }^{345}$

Es bleibt also unter Goldman, Bealer und Kornblith vieles umstritten. Hinsichtlich der Frage, ob Philosophinnen und Philosophen mittels der Intuition mentale Entitäten oder direkt die Außenwelt untersuchen, besteht der Gegensatz Goldman vs. Bealer und Kornblith. Hinsichtlich der a priori/a posterioriFrage hingegen verläuft die Front zwischen Goldman und Bealer vs. Kornblith. Ungeachtet dieser Gegensätze geben Goldman, Bealer und Kornblith gemeinsam der Intuition eine zentrale Bedeutung für die philosophische Arbeit.

Die Intuition als Erkenntnisquelle hohen Werts einzuordnen war auch das Bestreben der vorliegenden Untersuchung. Daneben weichen die Ergebnisse dieser Untersuchung jedoch in verschiedenen Punkten von Goldmans, Bealers und Kornbliths Ansichten ab.

Erstens stellt sich die Frage, ob das hier erarbeitete Intuitionsverständnis zur Unterstützung des Mentalismus oder des Extramentalismus passt. Hier ist zunächst Letzteres zu befürworten: In dieser Arbeit wurden Intuitionen in die epistemologische Untersuchung aufgenommen, welche der Mentalist Goldman scharf von philosophisch respektablen Intuitionen trennt und im Gegensatz zu diesen als garden-variety intuitions bezeichnet. ${ }^{346}$ Philosophische Intuitionen sind Goldman zufolge Anwendungsintuitionen; das heißt, es geht darum zu prüfen, ob ein Fall zu einem gegebenen Begriff passt, worüber die Intuition Aufschluss gibt. Die Kritik an Goldmans Ansatz, wonach Intuition nicht auf diese spezifische Begriffsanwendungs-Situation eingegrenzt werden sollte, ist auch aus der Perspektive des in dieser Arbeit vertretenen Intuitionsverständnisses plausibel. Trotz der Ablehnung des Mentalismus soll aber eingeräumt werden, dass sich Intuition, sei es in allgemeinen oder in philosophischen Erkenntniszusammenhängen, oft auf die Anwendung von Begriffen bezieht. Auf Begriffsanwendung bezogene Intuition zeigt sich dabei kaum weniger fehleranfällig als andere Formen der Intuition. Dies zeigen etwa Befunde des psychologischen Essentialismus ${ }^{347}$ und stereotypisierende oder gar rassistische Intuitionen, welche die Gefahren unreflektierter intuitiver Begriffsanwendung deutlich werden lassen.

345 Goldman (2007, S. 8); siehe andererseits auch Kornbliths Replik und Kritik an Goldmans Ansatz: Kornblith (2007).

346 Vgl. Goldman (2007, S. 3).

347 Vgl. Gelman (2003). 
Ein zweiter Gegensatz zwischen den genannten Positionen wurde durch die Frage bestimmt, ob sich philosophische Intuitionen ausschließlich auf den Bereich des Apriorischen richten, wie dies George Bealer mit seinem modalen Intuitionsverständnis postuliert. Hier ist die Positionierung zu einem großen Teil bereits vorgenommen worden: Zugunsten eines grundlegenden Intuitionsverständnisses ist der Apriorismus im Hinblick auf intuitive Erkenntnis abzulehnen. Zum einen geht es um Intuition als Erkenntnisgattung und um das erkenntnistheoretische Verständnis dieser Intuition, wofür der Apriorismus zu eng ist. Zum anderen wurde durch den Einbezug alltäglicher Erfahrungen und wissenschaftlich-empirischer Befunde unweigerlich gegen einen philosophiemethodischen Apriorismus Stellung bezogen. Obwohl begriffliche Arbeit, etwa die Untersuchung logischer Zusammenhänge, in der Philosophie zentral ist, wäre doch kaum Interessantes über Intuition als grundlegende Erkenntnisart auszumachen gewesen, wenn nicht alltägliche und wissenschaftliche Erfahrung einbezogen worden wäre.

Drittens bleibt die naturalistische Intuitionsauffassung, also spezifisch Kornbliths Position, aus Sicht des hier erarbeiteten Intuitionsverständnisses zu beurteilen. Hierbei ist zunächst positiv festzuhalten, dass Kornblith die etwa bei Bealer ausgeklammerten physischen Intuitionen wieder aufnimmt, indem er den Apriorismus grundsätzlich zurückweist. Dieser Inklusion folgt auch die vorliegende Studie, indem hier der Anspruch erhoben wurde, Intuition als Erkenntnisart in ihrer Breite zu erfassen. Hierfür wurden auch empirische Belege einbezogen, was einer Forderung Kornbliths entspricht. ${ }^{348}$ Als problematisch ist jedoch ein anderer Punkt in Kornbliths Intuitions-Naturalismus zu benennen. Kornblith fixiert den Anwendungsbereich der Intuition auf natural kinds. In naturalistischer Auffassung sind auch allgemeine Konzepte wie etwa >Wissen natural kinds. Nach dieser Auffassung hat Intuition einen rein heuristischen Wert:

If my account is correct, then what we ought to be doing is not just consulting the beliefs we already have, but more directly examining the external phenomena; only then would appeals to intuition given what, on my view, is their proper weight. Thus, appeal to intuition early on in philosophical investigation should give way to more straightforwardly empirical investigations of external phenomena. ${ }^{349}$

Dieses von Kornblith geforderte Primat der diskursiv-empirischen Untersuchung vor der intuitiven ist in Frage stellen. In dieser Arbeit wurde dafür

348 Vgl. Kornblith (1998, S. 136f.).

349 Kornblith (1998, S. 135). 
$\operatorname{argumentiert,~dass~Intuitionen~}_{\mathrm{NP}}$, also nichtbegriffliche Gehalte, Gründe für Subjekte sein können. Trifft dies zu, lassen sich daraus zwei Konsequenzen für die Philosophiemethodik ableiten; eine schwächere und möglicherweise auch eine stärkere.

Die schwächere Konsequenz zielt auf die Ebenbürtigkeit zwischen intuitiver und empirischer Erkenntnis ab. Der Appell an die Intuition, etwa herbeigeführt durch Beispiele, kontrafaktische Szenarien, Gedankenexperimente oder den erweiterten Einbezug fiktionaler Formen, wird aufgrund dieser Sichtweise zur berechtigten und wertvollen philosophischen Technik. Es gibt keinen Grund, auf solchen Prozessen beruhende Erkenntnisse erst nach einer vollständigen Diskursivierung oder gar erst nach einer Überantwortung an empirische Wissenschaften gelten zu lassen, da intuitives Denken als grundsätzlich gleichwertig zum diskursiven Denken anerkannt wird, sofern es nachvollziehbar ist. Die Nachvollziehbarkeit kann gerade durch die erwähnten Beispiele, Gedankenexperimente usw. gewährleistet werden, da diese darauf abzielen, bestimmte Intuitionen auch bei den Rezipienten in Gang zu setzen.

Die Untersuchungen zur intuitiven Erkenntnis und Rechtfertigung lassen auch einen stärkeren Schluss zu: Letztlich beruht jede philosophische Argumentation, auch eine solche, die diskursive oder empirische Erkenntnisse einbezieht, auf Intuition. Dieser Einsicht sind wir bereits bei Descartes begegnet: In einer deduktiven Kette muss der Übergang zwischen jedem Glied der Kette intuitiv gesichert sein. Zumindest auf diese Weise muss auch in der Philosophie Intuition das Fundament aller Erkenntnis sein. Eine weitere Weise der intuitiven Fundierung könnte darin bestehen, dass wir bei aller Argumentation immer bestimmte Prämissen als gegeben annehmen müssen. Letztlich kann daher jeder Beginn einer philosophischen Argumentation als Versuch gesehen werden, an einen common ground zu appellieren, von dem aus die gewünschten Schlüsse gezogen werden. Die Stützung dieser Prämissen bzw. des common ground erfolgt oft via Intuition, da sich eben die diskursive Untersuchung nicht endlos weiter erstrecken kann. Die Argumentation als Kern der philosophischen Methode kann also in einem oder gar beiden der erwähnten Sinne nicht ohne Intuition vonstattengehen.

Zusammenfassend befindet sich meine Position mit den Ansätzen Goldmans, Bealers und Kornbliths im Hinblick auf deren Hochschätzung der Intuition als Quelle philosophischer Erkenntnis in Übereinstimmung. Im Einzelnen ergeben sich jedoch Einwände gegen alle drei Positionen. Eine Neuorientierung der philosophiemethodischen Debatte ist wünschenswert, die das Primat der erkenntnistheoretischen Fragen in Bezug auf Intuition anerkennt und die methodischen Fragen darauf aufbauend behandelt. Die im Folgenden besprochenen Ansätze Stichs und der experimentellen Philosophie 
sowie Williamsons liefern wertvolle Impulse für eine solche Neuorientierung, obgleich sie andere Probleme aufweisen.

\subsection{Experimentelle Philosophie: Die Intuitionskritiker}

Stephen Stich vertritt wie Kornblith eine naturalistisch orientierte Erkenntnistheorie. Im Gegensatz zu Kornblith stellt Stich jedoch die Intuition als philosophische Methode grundsätzlich infrage. ${ }^{350}$ In Bezug auf die Gettier-Fälle argumentiert Stich wie folgt:

once it is seen that the notion we happen to have inherited is but one among many possible alternative notions, most people are not much inclined to say that they find having justified beliefs to be intrinsically valuable. Since our notion of justification is just one member of a large and varied family of concepts of epistemic evaluation, it strikes most people as simply capricious or perverse to have an intrinsic preference for justified beliefs. ${ }^{351}$

Im selben Zusammenhang wirft Stich Philosophen, die glauben, ihre Intuitionen haben universelle Gültigkeit, »epistemischen Chauvinismus « vor. ${ }^{352}$ Der Vorwurf des Chauvinismus richtet sich gegen eine Erkenntnistheorie, die sich unreflektiert auf angeblich universelle Intuitionen beruft um ihre Thesen zu rechtfertigen und dabei verkennt, dass diese Intuitionen möglicherweise nicht universell sind.

Was Stich in seinen früheren Arbeiten noch als Möglichkeit darstellt, versucht er später gemeinsam mit Jonathan Weinberg und Shaun Nichols empirisch nachzuweisen. Dies mündet im 2001 erschienenen Aufsatz Normativity and Epistemic Intuitions, der als Gründungstext der sogenannten experimentellen Philosophie gelten darf. ${ }^{353}$ In Weinbergs Studie wurden um die 200 US-amerikanische Studierende westlicher bzw. indischer Herkunft

$35^{\circ}$ Siehe das Kapitel Against Analytic Epistemology in Stich (1990, S. 89-100); sowie Stich (1998, S. 104-109).

351 Stich (1990, S. 95).

352 Vgl. Stich (1990, S. 99).

353 Siehe Weinberg/Nichols/Stich (2001). Seither haben sich Arbeiten der experimentellen Philosophie mit verschiedensten philosophischen Fragen befasst, zu deren Beantwortung eine Erhebung der tatsächlichen Intuitionen beitragen könnte. Die experimentelle Philosophie ist der Ansicht, dass viele philosophische Thesen empirisch überprüfbare Aussagen über tatsächliche Intuitionen machen. Solche Thesen sind folglich mit empirischen Mitteln falsifizierbar (vgl. Alexander 2012, S. 44f., sowie die ganze Monographie Alexanders zur Einführung in die experimentelle Philosophie). 
zu Gettier-Beispielen befragt. ${ }^{354}$ Es ergab sich den Autoren zufolge ein signifikanter Unterschied zwischen den beiden Gruppen der Befragten: Während die Subjekte westlicher Herkunft überwiegend Gettiers Intuition zustimmten, wonach die Person im Beispiel nur glaubt, das Fragliche zu wissen und es nicht wirklich weiß (wodurch die Platonische Wissensdefinition falsch wäre), war dies bei den Subjekten indischer Herkunft nicht der Fall. Eine Mehrheit der Befragten dieser Gruppe war in den beiden präsentierten Gettier-Fällen der Meinung, dass das Subjekt tatsächlich über Wissen verfügt. Ähnliche Unterschiede hinsichtlich der Gettier-Intuitionen zeigten sich zwischen Gruppen von Subjekten mit unterschiedlichem sozioökonomischem Status.

Weinberg, Nichols und Stich führen diese Resultate als Evidenz gegen eine Haltung, die sie als Intuition-Driven Romanticism (IDR) bezeichnen, ins Feld. Der IDR geht von epistemischen Intuitionen als Input aus und stellt normative Thesen zu epistemischen Fragen auf, die von den jeweiligen Intuitionen abhängig sind. ${ }^{355}$ Insofern IDR-Philosophinnen und -Philosophen unhinterfragt von bestimmten Intuitionen ausgehen und diesen teilweise gar apriorischen Status zubilligen, sind ihre Thesen unzureichend abgestützt, da die Intuitionen - wie im Gettier-Beispiel gezeigt - in unerwarteter Weise variieren können. Dem Lager der experimentellen Philosophie zufolge sollte sich die Philosophie vermehrt der Frage zuwenden, welche Intuitionen Menschen tatsächlich haben, statt kontingente Intuitionen unhinterfragt $\mathrm{zu}$ verallgemeinern.

Die Aussagen Stichs und Weinbergs haben zahlreiche Repliken hervorgerufen. Eine erste Strategie zur Verteidigung der Intuition als philosophische Methode besteht darin, sich auf die engen Intuitions- und Philosophieverständnisse zurückzuberufen. Diese Strategie stößt aber bald an ihre Grenzen. So scheint im Fall der Analyse von >Wissen< der Nachweis, dass die Gettier-Intuition nicht apriorische Gültigkeit haben kann, schlicht dadurch erbracht, dass diese offenbar nicht notwendigerweise im Zusammenhang mit den Gettier-Fällen auftritt. Als zweite Strategie wurde die empirische Qualität der Weinberg-Studie in Zweifel gezogen. Die Resultate von Replikationen der Studie deuten darauf hin, dass die epistemischen Intuitionen im Vergleich zwischen Gruppen mit unterschiedlicher Herkunft und sozioökonomischem

354 Genauer handelte es sich bei der zweiten Gruppe um Studierende mit Abstammung >indischer Subkontinent< (Indien, Bangladesch, Pakistan). Alle Studierenden besuchten die Rutgers-Universität im US-Bundesstaat New Jersey.

Vgl. Weinberg/Nichols/Stich (2001, S. 432). 
Status viel einheitlicher sind als von Weinberg befunden. ${ }^{356}$ Stichs Überlegung zur möglichen Relativität der Intuition bleibt aber plausibel, weshalb es besser wäre, wenn gezeigt werden könnte, dass die Relativität der Intuition diese als philosophische Methode nicht grundsätzlich in Frage stellt. Eine dritte Verteidigung des klassischen Intuitionsverständnisses besteht darin, auf das psychologisch fundierte Konzept der Expertenintuition zurückzugreifen. Demzufolge ist es irreführend, beliebige Subjekte nach ihren Intuitionen zu philosophischen Fragen oder Beispielen und Gedankenexperimenten zu befragen um daraus Rückschlüsse hinsichtlich der Gültigkeit der auf diesen Beispielen aufbauenden Positionen zu gewinnen. ${ }^{357}$ Hiergegen ist einzuwenden, dass auch Expertinnen und Experten große Fehler begehen können, wenn sie sich auf ihre Intuitionen berufen. ${ }^{358}$ Eine vierte Replik auf Weinberg ergibt sich, wenn der im Gettier-Beispiel gezeigte Dissens zwischen verschiedenen Gruppen als nicht-substantielle Meinungsverschiedenheit über die Bedeutung von Begriffen verstanden wird. ${ }^{359}$ Es mag sein, dass Subjekte Beispiele unterschiedlich verstehen und die unterschiedlichen Intuitionen darauf zurückzuführen sind. Dieser Punkt ist aber zu schwach, da er nur auf die Möglichkeit eines verbalen Dissenses hinweist. Es müsste vielmehr gezeigt werden, dass alle Intuitions-Dissense auf diese Weise auflösbar sind, was nicht erfolgt ist und auch nicht plausibel scheint.

Die angeführten Einwände gegen Weinbergs und Stichs Kritik der Intuition als philosophische Methode sind also nicht überzeugend. Die kritische Haltung der experimentellen Philosophie gegenüber einer Überhöhung der Intuition als philosophische Methode lässt sich auch vor dem Hintergrund des hier erarbeiteten Intuitionsverständnisses bestätigen. Jedoch ist gegenüber Weinberg und Stich eine Differenzierung vorzunehmen: Zwei Funktionen der Intuition, die in der philosophiemethodischen Debatte oft vermischt wurden, sollten auseinandergehalten werden: die argumentative und die epistemische Funktion.

Der Unterschied zwischen argumentativer und epistemischer Funktion der Intuition kann anhand eines Rückgriffs auf das Kekulé-Beispiel dargestellt werden. Die entscheidende Frage lautet hier: Könnte eine Studie analog zu derjenigen Weinbergs durchgeführt werden, die zeigt, dass Benzolring-Intuitionen

\footnotetext{
356 Vgl. Seyedsayamdost (2015).

357 Vgl. Hales (2006, S. 171).

$35^{8}$ Vgl. Weinberg et al. (2010).

359 Vgl. Sosa (2006a); Ludwig (2010).
} 
kulturell variieren, und dürfte daraus auf die Unzulänglichkeit der Intuition als (wissenschaftliche) Erkenntnisquelle geschlossen werden? Dies ist offenbar nicht der Fall. Der Grund dafür ist, dass das Resultat des intuitiven Erkenntnisvorgangs in Fällen wie demjenigen Kekulés entweder selbstevident oder zumindest nachprüfbar ist. Wir haben in diesen Fällen einen Vergleichspunkt - das Tatsachenwissen - anhand dem wir nachträglich oder als Außenstehende die Zuverlässigkeit der Intuition beurteilen können. Dieser Vergleichspunkt fehlt bei den Intuitionen bezüglich Wissen. Dies deutet darauf hin, dass Wissen kein natural kind ist, sondern Begriffe wie >Wissen< immer eine willkürliche und normative Komponente enthalten. Diese Begriffe mögen sich zwar auf Tatsachen in der Welt beziehen, aber wie sie diese einordnen (z.B., nach welchen Kriterien die Resultate der kognitiven Prozesse als Fälle von Wissen gelten dürfen), sind Entscheidungen der Menschen, welche die Begriffe verwenden.

Somit ist es nicht überraschend, dass die Gettier-Intuitionen in Abhängigkeit von der kulturellen oder sozialen Zugehörigkeit der Subjekte variieren. Ähnliche Variationen sind unumstritten bei vielen ethischen Konzepten vorhanden, bei denen die Willkürlichkeit und Normativität der Begriffsbildung nur offensichtlicher ist als beim Wissensbegriff. Die Weinberg-Studie lehrt uns also nichts über die Zuverlässigkeit der Intuition, sondern sie gibt uns bestenfalls einen weiteren Beleg dafür, dass willkürliche und normative Begriffe in unterschiedlichen kulturellen und sozialen Gruppen unterschiedlich verwendet werden. Tatsächlich wird dadurch die Auffassung, wonach man sich bei solchen Konzepten auf »felsenfeste« Intuitionen berufen könne um ihren Inhalt universell zu fixieren, widerlegt. Jedoch betrifft diese Widerlegung nicht die Intuition als Erkenntnisform. Vielmehr geht es um unterschiedliche und zudem normativ aufgeladene Begriffsverständnisse, die man ebenso im Bereich diskursiver Argumentationen antrifft. Nicht die Intuition ist also das »Problem«, sondern v.a. die unterschiedlichen mentalen Modelle, zu welchen die Intuition jeweils hinführt!

Durchaus spielt die Intuition bei der Studie Gettiers eine entscheidende Rolle; hierbei handelt es sich aber um die argumentative Funktion der Intuition. Gettier will mit seinen Beispielen die Lesenden oder Zuhörenden von der These überzeugen, dass die platonische Wissensdefinition falsch ist. Dazu appelliert er an eine gemeinsam geteilte Intuition als common ground, von dem aus die weitere Argumentation aufgebaut werden kann. Der Appell an die Intuition deutet hierbei entweder an, dass man weitere deduktive Schritte zur Herleitung des common ground nicht explizieren will (etwa, weil die Intuition als unkontrovers angenommen wird) bzw. nicht explizieren kann, oder dass 
die Gründe erst ex post gegeben werden sollen, nachdem die Lesenden via gemeinsamer Intuition bereits ins Boot geholt wurden. ${ }^{360} 361$

Aus dieser Diagnose zeichnet sich ab: Was eine philosophiemethodische Debatte zunächst braucht, ist eine Untersuchung der epistemischen Funktion der Intuition. Eine solche Untersuchung wurde in der vorliegenden Arbeit vorgenommen. Dabei wurde deutlich, dass durchaus Vorbehalte und Herausforderungen gegen die Intuition als Erkenntnisquelle bestehen können. Alle Arten kognitiver Fehlleistungen und biases, gegen welche das Typ 1-Denken besonders anfällig ist, sind hier zu nennen. Solche Vorbehalte sind folglich auch in der philosophiemethodischen Debatte ernst zu nehmen. In diesem Sinn braucht es Regeln, wann und in welcher Form sich Philosophierende auf Intuitionen stützen dürfen oder gar sollen.

Die Weinberg-Studie und Stichs Überlegungen sagen über diese epistemische Funktion der Intuition wenig aus. Vielmehr wird hier deutlich, dass unterschiedliche Verständnisse eines Begriffes vorliegen, die wohl größtenteils durch unterschiedliche mentale Modelle konstituiert werden. Dies ist auf der epistemischen Ebene nicht überraschend; wie es auch bei einem deduktiven Argument, bei dem die Prämissen nicht identisch sind nicht überrascht, dass die beiden Seiten nicht zur selben Konklusion kommen. Doch insofern Weinberg et al. gegen die Auffassung argumentieren, wonach uns Intuitionen zu felsenfesten und kulturunabhängigen Wahrheiten führen, ist ihnen sicher beizupflichten: Intuitionen können die ihnen zugrundeliegenden mentalen Modelle in der epistemischen Qualität nicht übertreffen.

\subsection{Der dritte Weg: Philosophie ohne Intuition}

Timothy Williamson, Herman Cappelen, Max Deutsch und andere vertreten die These, dass die bisherige Diskussion in die Irre ging, da die Intuition überhaupt keine, oder zumindest keine relevante, philosophische Methode konstituiert.

360 Letzteres ist auch bei Gettier der Fall. Er deutet an, dass die durch seine Beispiele evozierten Intuitionen daher rühren, dass das angebliche Wissen nicht die richtige kausale Vorgeschichte hat, um als genuines Wissen gelten zu können (vgl. Gettier 1963). Da hier - wie in vielen Fällen - die Intuition auf das eigentliche Thema bezogen ist und nicht bloß als Startpunkt für eine anderweitige Untersuchung gilt, darf dieses Explizitmachen der Gründe und damit das >Auflösen< der Intuition von einer guten philosophischen Arbeit erwartet werden.

361 Zum Zusammenhang zwischen Intuition und ex post-reasoning siehe die anthropologisch und kognitionspsychologisch fundierten Thesen in Mercier/Sperber (2017). 
Aus deren Perspektive gehen sowohl die Intuitions-Traditionalisten als auch die Intuitionskritiker Stich und Weinberg von der falschen Grundannahme aus, dass die Intuition bisher eine zentrale Rolle in der Philosophie innehatte. In Wahrheit spiele die Intuition keine oder nur eine marginale Rolle als philosophische Methode. Diese Ablehnung der Intuition als Erkenntnismethode in der Philosophie kann aus der Perspektive der vorliegenden Studie selbstverständlich nicht geteilt werden. Doch auch aus der Auseinandersetzung mit der Philosophie-ohne-Intuition-Position können wichtige Hinweise für ein vertieftes Intuitionsverständnis gewonnen werden.

\subsubsection{Williamson: knowledge first-Ansatz}

Timothy Williamson hat erste skeptische Überlegungen im Hinblick auf die Rolle der Intuition in der Philosophie angestellt. ${ }^{362}$ Im Folgenden sollen die Überlegungen Williamsons herausgearbeitet und thesenförmig dargestellt werden. Im Anschluss an die Thesen wird jeweils aus der Perspektive des hier vertretenen Intuitionsverständnisses Stellung genommen.

(1) Man bemerkt introspektiv schlicht keine Intuition. ${ }^{363}$

Dies ist die absent intuition challenge, wie Chudnoffs treffende Bezeichnung dafür lautet. ${ }^{364}$ Williamson richtet diese Kritik gegen Bealers Position, der die Intuition als intellektuelle Erscheinung versteht:

For myself, I am aware of no intellectual seeming beyond my conscious inclination to believe the Gettier proposition. Similarly, I am aware of no intellectual seeming beyond my conscious inclination to believe Naïve Comprehension, which I resist because I know better. ${ }^{365}$

Williamson wendet gegen Bealers Intuitionsverständnis ein, dass der intellektuellen Erscheinung die reichhaltige phänomenale Ebene einer perzeptiven Erscheinung fehlt und es schon deshalb fragwürdig ist, mit der Erscheinungsanalogie zu arbeiten. Darauf aufbauend insistiert Williamson, in den fraglichen Fällen keine Intuition (mit der postulierten Phänomenologie) zu haben.

Wird die Kritik Williamsons in enger Weise als Kritik an Bealers Sicht verstanden, kann man ihr vermutlich zustimmen. Williamsons gegen Bealer vorgenommene Beschreibung der Intuition als Neigung zu einer Überzeugung wurde oben auf ähnliche Weise vertreten (Kap. 3.2). Will Williamson aber der

362 Siehe Williamson (2004, 2007).

363 Vgl. Williamson (2007, S. 216-218).

364 Vgl. Chudnoff (2013, S. 52-57).

365 Williamson (2007, S. 217). 
Intuition die Phänomenalität ganz absprechen, ist ihm zu widersprechen. Wenn Williamson von einer »conscious inclination to believe« spricht, hat er die Schwelle für ein phänomenales Erleben bereits überschritten; denn bewusst zu erleben, dass man eine Neigung zu einer Überzeugung hat, weist sicherlich eine phänomenale Qualität auf, wenn auch eine von der Wahrnehmung verschiedene. Zu dieser phänomenalen Qualität gehören maßgeblich Unmittelbarkeit und Gewissheit (siehe Kap. 3.1).

(2) Nur der Gehalt einer Intuition (intuited) kann als Evidenz dienen, nicht das Haben der Intuition (intuiting). ${ }^{366}$

An Williamsons Ablehnung einer sui generis-Intuitionskonzeption ist seine Sichtweise geknüpft, dass Philosophinnen und Philosophen oft herkömmliche Gründe oder Überzeugungen als intuitiv bezeichnen, wobei sie diesen Zusatz in epistemologischer Hinsicht genauso gut weglassen könnten. Als Beispiel führt Williamson den lächerlich wirkenden Ausspruch eines Philosophieprofessors an, der anlässlich einer Konferenz als Argument für die These, dass Personen nicht identisch mit ihren Gehirnen sind, äußert: »Ich habe die Intuition, dass ich mehr wiege als drei Pfund.«Williamson zufolge meint der Professor einfach, dass er dies wei $\beta .{ }^{367}$ Wenn man überhaupt von Intuitionen sprechen will, ist es demnach ausschließlich der Gehalt der Intuition (intuited), der einen Grund, etwa für eine andere Überzeugung, darstellt. Nun beobachtet Williamson, dass diese Auffassung von den kritisierten Philosophen nicht geteilt wird; vielmehr wollen sie offenbar das Haben der Intuition (intuiting) als Teil der Evidenz gelten lassen. ${ }^{368}$

Das Modell der intuitiven Rechtfertigung, das in dieser Arbeit skizziert wurde, integriert - konträr zu Williamson - das intuiting explizit in die Evidenz: Eine der Bedingungen dafür, dass eine Intuition Grund für ein Subjekt ist, ist nach diesem Modell, dass das Subjekt durch Bekanntschaft und mentale Demonstration seine Intuition als solche einzuordnen vermag (siehe Kap. 4.5.3). Dass es sich um eine Intuition handelt, ist dem Subjekt somit bewusst. Dies ist entscheidend für die intuitive Rechtfertigung. Würde das Bewusstsein über den mentalen Zustand wegfallen und ließen wir nur den intuierten Gehalt als Evidenz zu, könnte man in zahlreichen Beispielen sehen, wie die Evidenz in sich zusammenfällt. Beispielsweise ist es entscheidend, dass der Gehalt >wenn die Feuerwehrmänner im Gebäude bleiben, sind sie in Lebensgefahr nicht etwa der Gehalt einer Imagination, eines Wunsches, einer Annahme oder einer vagen Vermutung, sondern der Gehalt einer Intuition ist,

366 Vgl. Williamson (2004, S. 119; 2007, S. 235f.). Siehe auch Langkau (2017, S. 13f.).

367 Vgl. Williamson (2004, S. 110; 2007, S. 214).

368 Vgl. Williamson (2007, S. 235f.). 
und dies muss dem Subjekt bewusst sein. Durch die intuitionsspezifischen phänomenalen Merkmale zeigt sich dem Subjekt an, dass es sich in diesem erkenntnisrelevanten (wenn auch falliblen) mentalen Zustand befindet. ${ }^{369}$

(3) Philosophinnen und Philosophen verweisen auf das Haben der Intuition als (erfolglose) Abwehrstrategie gegen den Skeptizismus (= Psychologisierung der Evidenz). ${ }^{370}$

»When contemporary analytic philosophers run out of arguments, they appeal to intuitions «, behauptet Williamson. ${ }^{371}$ Warum sie dies tun (wie der Philosophieprofessor im obigen Beispiel), erklärt Williamson mit dem Druck des Skeptizismus. Die Reaktion der von Williamson kritisierten Philosophen besteht nicht etwa darin, dem Urteilsskeptizismus zu widersprechen, sondern zuzugeben, dass wir uns zunächst nur über die Erscheinung sicher sein können. Sie suchen dem Skeptizismus aber dadurch zu entgehen, dass sie auf die Intuition als besondere Form dieser Erscheinungsevidenz verweisen. Diese Strategie ist Williamson zufolge erfolglos, weil unklar bleibt, warum eine psychologische Evidenz über die Intuition eines Subjekts eine Evidenz hinsichtlich der Wahrheit außerhalb dessen Psyche sein soll.

Williamsons strikter Trennung zwischen Evidenz über die Psyche und Evidenz über Tatsachen kann im Lichte der bisherigen Ausführungen nicht zugestimmt werden. Der Verweis auf die psychologische Ebene kann durchaus als Teil der Evidenz für außerpsychologisches Wissen gelten. Wenn wir die intuitive Rechtfertigung sowohl internalistisch als auch externalistisch untermauern können (was in dieser Arbeit versucht wurde), sind die Subjekte dazu berechtigt, vom Haben ihrer Intuition auf entsprechende Tatsachen in der Außenwelt zu schließen. Dass die Subjekte zunächst den Blick auf ihre eigene Kognition richten und bemerken, was sie erleben, ist dabei kein problematischer Umweg, sondern ein notwendiger Bestandteil der Evidenz. Die Intuition muss sich dem Subjekt als solche anzeigen, damit sie neben dem externalistischen das internalistische Erfordernis erfüllen kann, ein Grund für das Subjekt zu sein.

369 Auch aus dieser Perspektive wirkt die Äußerung des Professors in Williamsons Beispiel absurd. Die Unsinnigkeit der Äußerung »ich habe die Intuition, dass ich mehr als drei Pfund wiege « rührt aber einfach daher, dass diese Intuition, selbst wenn sie besteht, bei weitem überboten wird durch einen epistemischen Zustand, den wir nach allen (außer nach extrem skeptischen) Einschätzungen als $>$ Wissen $<$ bezeichnen. Somit verhält sich der Professor ähnlich wie jemand, der sagt: »Ich habe nachgezählt, dass ich zwei Hände habe.« Beispiele wie dieses sind somit zu grobschlächtig, um Williamsons These stützen zu können.

370 Vgl. Williamson (2004, S. 113, 118f.; 2007, S. 235f.).

371 Williamson (2007, S. 214). 
(4) Es gibt keine neutrale Evidenz. Was als Evidenz für ein bestimmtes Wissen gelten kann, ist erst post hoc klar. Der Verweis auf Intuition bringt daher die Diskussion meist nicht weiter. ${ }^{372}$

Williamson kritisiert das Prinzip der Evidenz-Neutralität. Diese Kritik baut auf dem knowledge first-Ansatz auf, für den Williamson auch in weiteren erkenntnistheoretischen Diskussionen bekannt wurde. ${ }^{373}$ Man stelle sich eine Debatte vor, bei der die eine Seite für Position A argumentiert, die andere für die A widersprechende Position B. Nun will der Vertreter von A seine Position stützen, indem er sie - etwa in Bezug auf ein Beispiel - als intuitiv plausibel bezeichnet. B muss nun nicht bestreiten, dass Position A intuitiv plausibel ist. Vielmehr wird B oftmals die intuitive Plausibilität zugeben, dann aber dafür argumentieren, dass A trotzdem falsch ist. Typischerweise liefert B hierbei eine Erklärung dafür, wie die falsche Intuition zustande kommt. Solche Argumentationsmuster sind etwa in der Körper-Geist-Debatte in der Philosophie des Geistes anzutreffen, wenn Monistinnen dualistische Intuitionen wegzuerklären versuchen. Diese Beispiele belegen Williamson zufolge die knowledge first-These, wonach es keine neutrale Evidenz gibt, auf der Wissen aufbauen kann. Vielmehr verlaufe der Prozess in der entgegengesetzten Richtung: Zuerst stehe für die Vertreterinnen und Vertreter unterschiedlicher Positionen fest, was sie wissen. Darauf aufbauend suche jede Seite nach Evidenz für ihre Position oder versuche, die von der Gegenseite angeführte Evidenz zu entkräften. Evidenz könne somit immer nur relativ zu Wissen bestehen.

Kognitionswissenschaftliche Befunde stützen Williamsons These, dass Begründung und somit auch die Benennung von Evidenz in der Regel (oder gar immer) post hoc erfolgt. ${ }^{374}$ Die Reihenfolge `Wissen vor Evidenz« spricht jedoch nicht gegen die Intuition als zentrale Erkenntnismethode, sondern gerade für sie. Wissen kann zwar vor der Benennung der Evidenz bestehen, muss jedoch zu einem früheren Zeitpunkt erworben worden sein. Wenn diese Annahme zutrifft, muss es eine Erklärung dafür geben, wie das Wissen jeweils erworben wurde, wenn auch nicht via diskursives Denken ausgehend von einer neutralen Evidenz. Gemäß den kognitionswissenschaftlichen Befunden im Rahmen der Dual-Prozess-Theorie ist es das Typ 1-Denken, welches diese Rolle der Wissenserschließung in den meisten Fällen einnimmt; und genau in diesem $\mathrm{Zu}-$ sammenhang wurde die Intuition - als nichtbegriffliche Gehalte verbindende Erkenntnisform - verortet. Williamson scheint Evidenz ausschließlich als benennbare Evidenz zu verstehen. Dadurch erfasst er nur die post hoc-Seite der

372 Vgl. Williamson (2007, S. 212f.).

373 Siehe u.a. Williamson (2013).

374 Vgl. Mercier/Sperber (2017). 
Evidenz. Wenn wir den Blick aber auf die Typ 1-Prozesse richten, können die hier resultierenden Intuitionen $\mathrm{NP}_{\mathrm{NP}}$ durchaus als Evidenzen bezeichnet werden, wenn auch ihre Benennung als solche erst post hoc erfolgen kann.

Zusammenfassend kann festgehalten werden, dass Williamsons knowledge first-Ansatz die zentrale Stellung der Intuition für die Erkenntnis, gerade auch in der Philosophie, nicht infrage zu stellen vermag.

\subsubsection{Cappelen: Philosophy Without Intuitions}

In seiner Monographie Philosophy Without Intuitions bestreitet Herman Cappelen die These, wonach Philosophinnen und Philosophen bei ihrer Arbeit zentral auf Intuition als Evidenz zurückgreifen (kurz: >Intuitionsthese $<$ ). ${ }^{375}$ Cappelens Argumentation gegen die Intuitionsthese verläuft in zwei Teilen. Im ersten Teil untersucht Cappelen anhand von Textbelegen, wie Philosophen Wörter wie >Intuition $<$ oder >intuitiv $<$ in ihren Texten verwenden und kommt dabei zum Schluss, dass die Philosophen mit diesen Begriffen nicht auf Intuition als eine Quelle der Evidenz referieren. Im zweiten Teil untersucht Cappelen einige einschlägige philosophische Argumentationen, die gemeinhin als Belege für die Intuitionsthese angenommen werden; darunter Gettiers Kritik an der klassischen Wissensdefinition und Keith Lehrers truetemp-Beispiele zur Widerlegung des epistemologischen Externalismus. Hier geht es nicht mehr um den tatsächlichen Wortgebrauch, sondern um die argumentative Praxis. Cappelens Fazit in Bezug auf diese Beispiele ist ebenfalls negativ: Es werde in diesen Beispielen - entgegen der weit verbreiteten Annahme - gar nicht auf Intuition als Evidenz rekurriert, sondern argumentiert, indem Gründe für die jeweiligen Thesen angegeben werden. Dies lässt Cappelen zum Schluss kommen, dass herkömmliche Argumentation das Geschäft der Philosophinnen und Philosophen ist, ohne dass sie sich dabei auf Intuitionen berufen.

Die erste Argumentation Cappelens ist sicherlich weniger stark als die zweite. ${ }^{376}$ Eine Analyse philosophischer Texte hinsichtlich der Verwendung des Begriffs >Intuition< mag erhellend sein und auch zu berechtigter Kritik an ungenauen Verwendungen dieses Begriffs führen. Doch muss man sich vor Augen halten, dass sich philosophische Texte auch dann auf Intuition als Evidenz berufen können, wenn dies nicht explizit ausgedrückt wird; so etwa Platons Dialoge, in denen Gegenbeispiele gegeben werden, um eine bestimmte Definition zu hinterfragen. Nun ließe sich möglicherweise bestreiten, dass sich Platon und andere in diesen Beispielen auf Intuition als Evidenz berufen. Dies

375 Vgl. Cappelen (2012).

376 Vgl. Nado (2016, S. 793). 
entspricht jedoch bereits dem zweiten Argument Cappelens, welches sich auf die argumentative Praxis der Philosophie bezieht.

Cappelen bestreitet, dass sich Philosophen wie Gettier und Lehrer tatsächlich auf Intuitionen als Evidenz berufen. Vielmehr, so Cappelen, argumentieren sie im herkömmlichen Sinn. Betrachten wir dies kurz anhand von Lehrers truetemp-Szenario. ${ }^{377}$ Lehrer ruft die Lesenden dazu auf, sich einen Mann vorzustellen, in dessen Hirn - von ihm unbemerkt - ein sehr zuverlässiges Temperaturmessgerät implantiert wurde. Dieses Gerät lässt ihn zu wahren Überzeugungen bezüglich der aktuellen Temperatur kommen, die aus externalistisch-reliabilistischen Perspektive auch gerechtfertigt sind. Man nimmt weiter an, der Mann hinterfrage seine Überzeugungen nicht. Die Frage ist nun, ob der Mann in der jeweiligen Situation wirklich weiß, wie warm es ist. Anzunehmen ist, dass dies intuitiv verneint wird. Somit wäre der Reliabilismus unter Berufung auf die Intuition als Evidenz widerlegt. Dies ist die klassische Lesart, die Cappelen nun bestreitet. Er weist auf eine Aussage Lehrers hin, die direkt an dessen Darstellung des truetemp-Szenarios anschließt:

More than possession of correct information is required for knowledge. One must have some way of knowing that the information is correct. ${ }^{378}$

Cappelen analysiert diese Aussage wie folgt:

Since Mr. Truetemp has no way of knowing that the information is correct, he doesn't know. In the text, the negative answer to the question, »Does he know that it is?, « is derived from this more general principle. So the answer is not presented as a basic, unjustified but justifying starting point in argumentation. ${ }^{379}$

Denselben Punkt macht Cappelen in Bezug auf eine Vielzahl weiterer Beispiele. ${ }^{380}$ Es geht hier nicht um eine Zurückweisung seiner Analyse des truetemp-Beispiels im Einzelnen, sondern um die generelle Aussage, wonach die jeweiligen Beispiele oder Szenarien nicht dazu dienen, Intuitionen wachzurufen, die als Evidenz gebraucht werden, sondern diese lediglich herkömmliche deduktive Argumentationen einleiten oder allenfalls illustrieren.

Aus der Perspektive des hier vertretenen Intuitionsverständnisses ist Cappelens Position vor allem aus dem folgenden Grund zu kritisieren: Cappelen sieht Intuition und Argumentation als gegensätzlich zueinander,

377 Vgl. Lehrer (2000).

378 Lehrer (2000, S. 188).

379 Cappelen (2012, S. 168).

38o Vgl. Cappelen (2012, S. 130-187). 
was nicht zutrifft. Der Verweis auf Intuition ist, wie bereits angedeutet wurde, eine Form der Argumentation. Um diesen Gedanken zu veranschaulichen, kann man sich die Situation des Philosophieunterrichts vorstellen, in dem solche Beispiele und Szenarien wie dasjenige Lehrers gegeben werden. Nehmen wir an, das Beispiel ist so überzeugend, dass die ganze Klasse mit der Ansicht übereinstimmt, es handle sich hier nicht um Wissen. Damit ist - im Diskursraum des Klassenzimmers - die externalistisch-reliabilistische Theorie widerlegt. Es wird keine weitere Erklärung dafür benötigt, warum der Mann im Beispiel über kein Wissen verfügt. ${ }^{381}$ Dieser Punkt lässt sich für alle Fälle generalisieren, in denen eine Theorie oder Hypothese durch ein Gegenbeispiel oder -szenario kritisiert wird. Es handelt sich jeweils um eine Falsifikation, die bereits durch den intuitiven Schluss aus dem Beispiel oder Szenario erfolgt: Die Theorie sagt etwa in Bezug auf das truetemp-Beispiel voraus, dass der Mann die Temperatur kennt; denn die Voraussetzungen für Wissen gemäß Theorie sind im Szenario erfüllt. Intuitiv, d.h. unmittelbar und mit Gewissheit, scheint uns Lesenden aber klar, dass man dem Mann kein Wissen zuschreiben kann. Wie das Beispiel des Philosophieunterrichts zeigt, ist dieses methodische Vorgehen als erster Schritt zur Kritik einer Theorie oder These gängige Praxis und ist auch in den von Cappelen analysierten Beispielen als erster Schritt zu bezeichnen; unabhängig davon, dass die Philosophinnen und Philosophen in aller Regel zum zweiten Schritt übergehen und die Gründe für die Intuition explizit zu machen suchen.

Der Vorgang ist auf der Ebene der kognitiven Prozesse wie folgt plausibel zu beschreiben: Ein Subjekt wird mit einem Szenario inklusive einer Frage konfrontiert. Diese Frage löst einen kognitiven Prozess auf der Typ 1-Ebene aus, der zum Abrufen des mentalen Modells <Wissen $>$ führt. Mentale Modelle enthalten Voraussetzungen, Bedingungen, mögliche Folgen und viele weitere logische Bezüge. Im mentalen Modell für $<$ Wissen $>$ ist daher das, was später im Grund für die Intuition explizit gemacht wird, enthalten. Das mentale

381 Einen damit verwandten Kritikpunkt bringt David Chalmers an:

Cappelen stresses a number of times that these texts often differ in surprising ways from the way their arguments are described by later philosophers and by textbooks. But insofar as there is a difference here, it is presumably the arguments as represented in later texts and textbooks that are most influential and most central to the practice of philosophy.

(Chalmers 2014, S. 539)

Aus Chalmers' Gedanke lässt sich erkennen: Beim Sich-Berufen auf Intuition geht es um eine argumentative Praxis, nicht um eine statische Evidenz. Es ist davon auszugehen, dass in diesen späteren Lehrbüchern oft - oder zumindest zunächst, im Sinn einer didaktisch sinnvollen Gliederung - die in Bezug auf das Szenario hervorgerufene Intuition als zentral dargestellt wird, analog zum beschriebenen Vorgehen im Philosophieunterricht. 
Modell für $<$ Wissen $>$ enthält »One must have some way of knowing that the information is correct « (nicht notwendigerweise begrifflich repräsentiert) als notwendige Bedingung für Wissen. Aus dem gegebenen Szenario und $<$ Wissen> leitet das Typ 1-Denken einen Gehalt ab, der das Nichtwissen des Mannes repräsentiert. Dieser Gehalt tritt, hervorgerufen durch die explizite Frage »Weiß der Mann das?«, als intuitive Überzeugung ins Bewusstsein. In einem weiteren Schritt können Teile von $<$ Wissen $>$ explizit gemacht werden. Der Zugang zu $<$ Wissen $>$ wurde aber erst durch die Intuition in Bezug auf das Szenario möglich und bleibt an diese Intuition zurückgebunden. Lehrer beruft sich somit in der philosophischen Diskussion auf ein geteiltes Wissensverständnis, also auf ein unter den Gesprächspartnern in relevanten Teilen ähnliches mentales Modell $<$ Wissen $>$. Dies ist der common ground, der hier abgerufen wird, um die Gesprächspartner oder Lesenden von einer These argumentativ zu überzeugen. ${ }^{382}$

»Must have some way of knowledge« ist daher nicht ein Argument für Lehrers These, wie Cappelen dies sieht, sondern dient dem Explizitmachen einer bereits vorhandenen Intuition. Dies lässt sich abschließend auch so darstellen: Niemand wird von »must have ...« überzeugt sein, wenn diese Aussage nicht dem bereits intuitiv abgesicherten common ground, entspricht oder aber argumentativ auf einen anderen common ground zurückgeführt wird. In den von Cappelen analysierten Beispielen, die alle demselben Muster wie das truetemp-Beispiel folgen, behält die Intuition somit ihren zentralen Platz in der philosophischen Methodik: als Erkenntnisart, die auf einen common ground zurückführt und dadurch eine Grundlage für die weitere Argumentation darstellt. ${ }^{383}$

382 Interessanterweise akzeptiert Cappelen diese Charakterisierung der philosophischen Debatte, indem auch er sagt, dass an einen common ground appelliert werden muss. Cappelen insistiert aber, dass dies nicht via Intuition erfolgen müsse:

All conversations have propositions in the common ground - propositions that are taken for granted among conversational participants. So, for example, when I talk to my brothers it is common ground that Eftang is located more than 1 mile outside Sandefjord. I can rely on this as a premise in reasoning without backing it up in any way.

(Cappelen 2012, S. 119)

Cappelen ist darin zuzustimmen, dass vielen Diskussionen Prämissen als common ground zugrunde liegen, die nicht durch Intuition erschlossen werden, wie sein Beispiel zeigt. Damit zeigt er aber nicht, dass dieser common ground nie via Intuition abgerufen wird. Somit bleibt völlig plausibel, dass in philosophischen Diskussionen die Berufung auf den common ground oft via Intuition erfolgt. In jedem Fall obläge es Cappelen zu zeigen, dass dies nicht der Fall ist (vgl. Nado 2016, S. 796).

383 Damit ist nicht gesagt, dass die philosophische Methodik auf die beschriebene Vorgehensweise fixiert ist. Möglicherweise und wahrscheinlich gibt es auch andere 


\subsubsection{Deutsch: The Myth of the Intuitive}

Eine ähnliche Argumentation wie Cappelen verfolgt Max Deutsch in The Myth of the Intuitive. ${ }^{384}$ Dies zeigt sich bereits in der Einleitung, in der Deutsch seine Position ausgehend vom Gettier-Beispiel skizziert:

Gettier offers a clear argument for his judgment about his thought experiment. He gives reasons for thinking that his Smith character does not know that the man who will get the job has ten coins in his pocket. [...] Gettier's argument for the judgment that Smith does not know is right there in his presentation of the 10 Coins Case, quoted above. It is hard to miss, actually. And it's a good argument too, which is why Gettier's refutation of the JTB theory of knowledge is widely regarded as just that - a refutation. ${ }^{385}$

Ausgehend von diesem Beispiel betont Deutsch, die Rolle der Intuition in der Philosophie sei bei weitem übertrieben worden, wodurch auch das Projekt der experimentellen Philosophie, welche mit Verweis auf die Relativität und Fallibilität der Intuition die klassische Philosophiemethodik kritisiert, mehr oder weniger obsolet werde. ${ }^{386} \mathrm{Um}$ Wiederholungen $\mathrm{zu}$ vermeiden, werde ich nur auf eines von Deutschs Argumenten eingehen, welches die Einwände Cappelens wesentlich zu ergänzen scheint.

Deutsch vertritt in Bezug auf Gettier und Kripke die Ansicht, dass diese sich in ihren einflussreichen Aufsätzen überhaupt nicht auf Intuition abstützen; dies sei ein weitverbreiteter, jedoch falscher Mythos. Vielmehr gelte:

The original presentations rather make it appear as though each argument is simply the rejection of a generalization of the form »All F s are G s« on the basis of a counterexample of the form »There is an F that is not a G.«There is certainly no explicit appeal to a premise of the form »It is intuitive that there is an $\mathrm{F}$ that is not a G.«387

Deutsch behauptet also, im Gettier-Beispiel und in analogen Fällen werde die Evidenz direkt durch das Gegenbeispiel gebildet und nicht durch Intuitionen. ${ }^{388}$ Als mögliche Quelle, die zu der aus seiner Sicht falschen Annahme über Intuitionen in der Philosophie führt, ortet er Formulierungen

philosophische Methoden, mit und ohne zentralem Einsatz der Intuition. Dies muss hier aber nicht weiter erörtert werden. Es reicht festzustellen, dass Diskursmuster wie das hier besprochene eine zentrale Stellung in der Philosophie einnehmen.

384 Deutsch (2015).

385 Deutsch (2015, S. xvi).

386 Vgl. Deutsch (2015, S. xvi).

387 Deutsch (2015, S. 45).

388 Vgl. Deutsch (2015, S. 74, 80). 
in den Originaltexten von der Art »Es ist klar, dass ...«, »es ist offensichtlich, dass ...«, o.ä. ${ }^{389}$ Hier schwingt ein bereits bei Williamson diskutierter Punkt mit: Williamson zufolge sollte nicht das Haben der Intuition Teil der Evidenz sein, sondern nur deren Gehalt. Deutsch verstärkt Williamsons Punkt dahingehend, dass die Rede von Intuition auch im Gehalts-Sinn keine Rolle mehr spielen soll, denn es gehe in Beispielen wie demjenigen Gettiers einfach um Tatsachen, die offensichtlich wahr und dadurch Evidenz für die jeweilige These sind.

Deutschs Argumentation ist wenig überzeugend, weil der Gedanke, wonach die intuitive Plausibilität einer Tatsache Teil der Evidenz für diese Tatsache ist, schlicht nichts Befremdendes hat. Dieser Punkt wurde bereits bei Williamson erörtert. Spezifisch in Bezug auf Deutschs Darstellung der Gettier-Fälle muss die Replik erweitert werden: Wie soll ein solches Beispiel bzw. Szenario an sich eine Evidenz darstellen? Das Szenario handelt von Männern mit Münzen in ihren Hosentaschen. Dies ist isoliert betrachtet keine Evidenz für irgendeine These über Wissen. Erst der Denkschritt, der uns Lesenden vor Augen führt, dass gemäß traditioneller Theorie einerseits von Wissen die Rede sein müsste, wir aber andererseits diese Zuschreibung im Beispiel nicht vornehmen möchten, führt dazu, dass dieses Beispiel in eine Argumentation gegen die Wissensdefinition eingespannt werden kann. Zwar ist Deutschs Aussage im obigen Zitat beizupflichten, dass es sich bei >Smith und Jones< um ein Gegenbeispiel zur auf dem Prüfstand stehenden Definition handelt. Was aber macht >Smith und Jones< zum Gegenbeispiel? Nur die Tatsache, dass wir in diesem Szenario die Wissenszuschreibung nicht vornehmen möchten! Dies ist eine von unserer Intuition handelnde Tatsache. ${ }^{390}$ Wie klar es uns scheint, dass das Subjekt im jeweiligen Beispiel das Entsprechende nicht weiß (nämlich intuitiv, also sehr klar), muss also durchaus als Teil der Evidenz gegen die klassische Wissensdefinition gelten.

Die Auseinandersetzung mit den Intuitionsskeptikern Williamson, Cappelen und Deutsch hat zu drei hauptsächlichen Einsichten geführt: Erstens ließ sich feststellen, dass das hier vertretene Intuitionsverständnis mit einer knowledge first-Position (Williamson) nicht prinzipiell in Konflikt steht, sondern dieser in gewisser Hinsicht entgegenkommt. Zweitens wurde bekräftigt, dass das Haben der Intuition sehr wohl ein wichtiger Teil der Evidenz ausmacht. Drittens wurde die Unterscheidung zwischen der epistemischen

389 Vgl. Deutsch $(2015$, S. 81).

390 Hier wird behauptet, die Intuition bestehe völlig unangefochten. Dies kann, wie gezeigt wurde, von Vertreterinnen und Vertretern der experimentellen Philosophie bestritten werden. 
und der diskursiven Funktion der Intuition bestätigt, wobei letztere oft darin besteht, einen common ground zu offenbaren; eine Aufgabe, welche die Intuition gerade in philosophischen Debatten, die auf grundlegende mentale Modelle wie $<$ Wissen $>$ zurückgehen, sehr gut erfüllen kann.

Die wichtigste Erkenntnis aus der Auseinandersetzung mit allen Positionen innerhalb der philosophiemethodischen Debatte bleibt, dass eine epistemologische Untersuchung der Intuition als Erkenntnisart einer methodischen Beurteilung derselben vorgelagert sein muss. Das Intuitionsverständnis und das Modell intuitiver Rechtfertigung, welche hier erarbeitet wurden, führen dementsprechend zu einer neuen Sicht auf die in der methodologischen Debatte vertretenen Positionen und Argumente. ${ }^{391}$

391 Die hier entwickelte Kritik an der bisherigen philosophiemethodischen Debatte weist Parallelen zur Argumentation Marcus McGahheys und Neil van Leeuwens auf, die auf der Basis eines kognitiv-psychologisch motivierten »Interpretationismus « den vorherrschenden »Mainstream-Propositionalismus« in der Intuitionsdiskussion kritisieren (vgl. McGahhey/van Leeuwen 2018). 